\title{
Adjustable IOLs-Disrupting the Refractive IOL Industry
}

\author{
David F Chang \\ Department of Ophthalmology, University of California San Francisco, San Francisco, CA, US
}

DOl: https://doi.org/10.17925/USOR.2017.10.02.95

A djustable intraocular lenses (IOLS) are uniquely poised to disrupt the refractive IOL arena for both cataract and refractive lens exchange patients. This is because they will not only improve refractive outcomes, but will dramatically enhance the patient experience as well. Patients will be able to postpone confusing decisions about refractive targets and objectives until they can preview them postoperatively. The unique benefit of being able to customize and "choose" their pseudophakic vision after the surgery will be understandable, desirable, and easily explained to family and friends.

\section{Keywords}

Adjustable IOL, refractive surgery, cataract surgery, toric or presbyopia correcting $\mathrm{IOL}$, bilateral simultaneous surgery

Disclosure: David F Chang is a consultant for RxSight, Powervision, J\&J Vision, and Zeiss. No funding was received in the publication of this article. This article is a short opinion piece and has not been submitted to external peer reviewers.

Authorship: All named authors meet the International Committee of Medical Journal Editors (ICMJE) criteria for authorship of this manuscript, take responsibility for the integrity of the work as a whole, and have given final approval to the version to be published.

open Access: This article is published under the Creative Commons Attribution Noncommercial License, which permits any noncommercial use, distribution, adaptation, and reproduction provided the original author(s) and source are given appropriate credit.

Received: September 1, 2017

Piblished Online: October 20, 2017

Citation: US Ophthalmic Review, 2017;10(2):95-8

Corresponding Author: David F Chang, 762 Altos Oaks Drive, Los Altos, CA 94024, US. E: dceye@earthlink.net
Harvard Business School professor Clayton Christensen popularized the concept of disruptive innovation to explain why the most successful companies eventually fail. While the leading companies continue to develop better and more expensive technology, they fail to anticipate a sea change in the way new technology will be used and incorporated into their business or field-the disruptive innovation. I believe that adjustable intraocular lenses (IOLS) are going to be the technology that ultimately disrupts the field of refractive IOL surgery for both cataract and refractive patients alike.

Because both presbyopia and cataracts are normal aging conditions, the majority of patients eventually become refractive IOL candidates. Surgeons and their patients will welcome the improvement in refractive outcomes that adjustable IOLs will enable. Equally important, however, is how adjustability will transform the patient's refractive surgical experience-how patients will decide what specific refractive outcome they want and how we will provide this to them. This is how adjustable IOLs are poised to disrupt the competition.

\section{Why have refractive IOL adoption rates been disappointing for the past two decades?}

Although they have been available in the US since the mid-1990s, adoption of multifocal and even toric IOLs has been surprisingly low. That the additional costs are not covered by insurance is not the only significant barrier to growth. Additional factors include: (1) our inability to deliver LASIK-like refractive outcomes, (2) side effects from diffractive presbyopia correcting IOLS, (3) lack of surgeon confidence in being able to satisfy most patients, (4) patient difficulty in understanding the value proposition, (5) lack of patient word-of-mouth endorsement, and (6) lack of referring doctor promotion of premium IOLS.

Imagine if we could not enhance our LASIK patients and only had one "shot" at hitting emmetropia. Patient satisfaction, surgeon confidence, optometric endorsement, and positive patient testimonials would all suffer. However, many thousands of refractive IOL patients are left with tolerable but residual refractive error because the majority of cataract surgeons do not also perform LASIK. We all dread facing patients who are disappointed with their refractive outcome or are distraught by pseudophakic halos and starbursts. When technically perfect surgery was performed, this is particularly demoralizing and reduces surgeon confidence in recommending presbyopia correcting IOLs. Many optometrists who follow these dissatisfied patients over time develop a bias against multifocal IOLS. Predicting preoperatively which patients might be more forgiving as opposed to demanding or picky about their eyesight is an unreliable art. Even when patients are delighted and pleased following their surgery, they do not know how much of the benefit is due to the cataract removal versus their choice of IOL. Finally, ophthalmologists are perplexed by the frequent poor 
correlation between surgical anatomic success, residual refractive error, and subjective "20-happy" outcomes.

To gain wide popularity, a refractive procedure or technology must consistently generate high satisfaction by all stakeholders-patients, surgeons, and referring optometrists-along with fair and proper alignment of financial incentives. The procedure should consistently meet or exceed patient expectations, and generate "wow" experiences about which patients will brag to their friends. LASIK satisfies all three stakeholders in these ways and benefits from a brand name that the public recognizes and remembers. However, a crucial component of LASIK's success is our ability to enhance the initial refractive result.

Unlike with LASIK, we cannot simply tell refractive IOL patients that they will never need eyeglasses again. Instead, our cataract patients must struggle to understand concepts such as focal point, depth of focus, contrast sensitivity, astigmatism, hyperopia, myopia, and presbyopia. Comprehending these optical concepts becomes fundamental to understanding the value proposition of reducing, but not eliminating spectacle dependence. Patients are much less likely to select more expensive options if they do not fully understand the benefits, and this hinders adoption of toric IOLS despite their lack of optical trade-offs.

\section{What adjustable IOL technologies are in the developmental pipeline?}

RxSight (formerly Calhoun Vision) developed the first light adjustable lens (RXLAL). The 3-piece RXLAL includes diffusible, photosensitive silicone macromers that are dispersed in the overall silicone matrix. Cataract surgery with RXLAL implantation is performed using standard techniques. Approximately 3 weeks later, the patient is refracted and a slit lamp based digital light delivery device (LDD) system is used to deliver the ultraviolet (UV) light in a precisely programmed pattern to induce a predictable change in the shape and refractive power of the optic. Treatment times range between 60-120 seconds. After the newly adjusted refraction is confirmed several days later, a "lock-in" dose is given with the LDD to polymerize all remaining macromer, at which point no further refractive change will occur. Patients wear special UV-blocking spectacles until the lock-in step is completed, after which they are no longer required.

As a phase II investigator, I implanted the first 16 RXLALs in the US. Patients readily understood the concept and were willing to temporarily wear UVblocking spectacles for the long term benefit. The slit lamp light adjustments were performed with a contact lens, much like a YAG capsulotomy, and were easily tolerated. Following implantation, adjustment, and lock-in, the result is an intracapsular, three-piece monofocal IOL, which avoids the long-term anatomic uncertainties associated with drastically different IOL designs. The RXLAL completed the US phase III trial in early 2016 and submitted its Premarket Approval (PMA) application to the US Food \& Drug Administration (FDA) later that same year.

Perfect Lens LLC is developing a novel technology called refractive index shaping (RIS) that can modify the refractive properties of an implanted IOL in situ with a femtosecond laser. RIS can be used to postoperatively modify spherical aberration, toricity, and even multifocality of an implanted IOL. Rabbit studies have provided in vivo proof of concept with maintenance of good modulation transfer function. Human clinical trials have not yet begun.

\section{With advances in biometry, IOL formulae, and intraoperative aberrometry, do we need an adjustable IOL?}

Although new formulae, improved biometry, and intraoperative wavefront aberrometry have incrementally improved our ability to select the optimal IOL sphere, cylinder, and axis, they are still simply predictions that improve the mean-our batting average — without eliminating the standard deviation. We still cannot preoperatively measure and must therefore estimate each individual eye's effective lens position, posterior corneal astigmatism, and surgically induced astigmatism. Instead of relying on predictions, adjustable IOLs will treat the stabilized postoperative refraction, and should appeal to anyone desiring a specific refractive target with a monofocal IOL. They would be especially valuable for eyes at greater risk for IOL power surprise, such as those with prior keratorefractive surgery, mature cataracts impeding optical biometry, rigid contact lens wear, atypical keratometry, and a very short or long axial length.

Even without these risk factors, an adjustable IOL would provide enhanced assurance against being one of the 15-35\% of patients that do not end up within $0.5 \mathrm{D}$ of the intended target. Imagine hypothetically that there were two different LASIK procedures-one that you could not enhance, and a second, more expensive procedure that would allow enhancements. Which would you choose to have? Would you take your chances with the less expensive alternative because the refractive results are usually good in most patients?

The RXLAL submitted for FDA approval in the US will allow adjustment of sphere and cylinder and I believe it will achieve the best outcomes for low to moderate astigmatism. Although mean surgically induced astigmatism can be determined, the standard deviation of this value is significant at approximately 0.5 D. Posterior corneal astigmatism is difficult to measure. The effect of either manual or femtosecond laser astigmatic keratotomy still varies with corneal rigidity and may regress unpredictably over time. In addition, although misalignment greater than 10 degrees is uncommon, we cannot predict or prevent smaller degrees of toric IOL rotation. With an adjustable IOL, predicting posterior corneal and surgically induced astigmatism will not matter because we will be treating the stabilized postoperative refraction long after any IOL rotation has already occurred. Although I currently employ corneal topography and wavefront aberrometry, swept-source optical coherence tomography (OCT) biometry, multiple advanced formulae, intraoperative aberrometry, and digital intraoperative axis localization, ophthalmology residents using only basic biometry should achieve better astigmatism outcomes with an adjustable IOL than I currently do using all of these technologies. This innovation will therefore disrupt what we currently do for preoperative and intraoperative testing.

\section{Disrupting the patient experience-choosing your vision postoperatively}

As the number of different IOL choices increases, surgeons are spending increasing amounts of preoperative chair time discussing refractive options and targets. These are difficult and anxious decisions for patients because the competing choices have different tradeoffs, and yet there is no way to try them out. It is challenging for preoperative patients to comprehend the difference between being plano or -1.00 , the difference between having $1 \mathrm{D}$ of astigmatism versus none, and the difference of 1 versus $1.5 \mathrm{D}$ of intentional anisometropia for mini-monovision. Patients must make these lifelong decisions prior to surgery, and after finally selecting a refractive 
objective, we then add the disclaimer that we cannot guarantee producing a specific refractive outcome. Most surgeons spend far more time on preoperative counseling than on the actual surgery. Having to decide preoperatively among confusing and expensive choices that they cannot preview or later easily reverse is a stressful experience for patients.

By solving these problems, adjustable IOLs will appeal to both patients and surgeons. Patients can wait until they are pseudophakic to have an optometrist demonstrate different options to them postoperatively using the phoropter, trial lenses, or disposable soft contact lenses. After sampling different refractive outcomes, patients can then "choose" their eyesight. We can demonstrate how much better vision without astigmatism would be. For patients whose priority is reading without glasses, we can simulate the difference between -1.50 or -2.50 . After they try it for a day with a SCL trial, we can create, reverse, and adjust pseudophakic mini-monovision. We can let patients choose the optimal amount of myopia for their near eye, and then confidently deliver that very outcome along with excellent distance acuity in the far eye. In recent American Society of Cataract and Refractive Surgery (ASCRS) Clinical Surveys, pseudophakic monovision was used much more frequently than presbyopia correcting IOLs. Adjustability and reversibility should make this strategy even more popular, and will avoid the risk of significant dysphotopsia with diffractive presbyopia correcting IOLS.

Although most pseudophakic patients are happy with 20/25 and 20/30 uncorrected distance acuity, adjusting away their residual refractive error might produce 20/20 or 20/15 uncorrected vision. This adjustment could be a "wow" experience for patients, surgeons, and referring optometrists. Although keratorefractive surgery can correct residual refractive error, it carries the psychological barrier of requiring another "surgery" with informed consent forms that describe some surgical risk. In addition, many cataract patients have dry eyes and many cataract surgeons do not perform keratorefractive surgery and may not have a partner who does.

The analogy of buying a dress or suit works nicely for explaining the concept of IOL adjustment and customization to patients. You can buy a suit off the rack at the department store, which allows you to try the next size up or down. This is how we fit soft contact lenses. You can buy your suit over the Internet based on a few measurements with the hope that it will fit. This is how we select your IOL for cataract surgery because we can only implant and try out one lens power. Finally, you could have a tailor make alternations once you are wearing the suit so that it is custom fit to your body. This would be like adjusting your lens after it is inside your eye.

\section{Extended depth of focus (EDOF)}

This IOL category is growing in popularity for pseudophakic presbyopia correction. The tradeoffs of true monofocal monovision, such as loss or reduction of binocularity and depth perception, are well known. One strategy is to enhance depth of focus by increasing total ocular spherical aberration (SA). Mini-monovision with EDOF optics may provide good near function while preserving binocularity and avoiding the optical compromises associated with diffractive optics. Of course corneal SA varies individually within the general population. RxSight can create EDOF optics postoperatively by precise alterations in SA. This can be done in a way that allows pseudophakic patients to preview several possible SA profiles, and to select the one that works best for their eye, based on their corneal aberrations. Of course, this additional indication will require a new FDA approval.
Once approved for this indication, I believe that mini-monovision with adjustable IOLs employing EDOF optics will also be a game changer for refractive lens exchange (RLE). This strategy should appeal to the large population of patients in their 50s and 60s who covet and can most afford a lifestyle-enhancing, presbyopia-correcting refractive procedure. Compared to current diffractive IOL technologies, this strategy using monofocal IOLs preserves the highest-quality vision and reduces optical tradeoffs, such as halos, glare, and reduced contrast sensitivity. This is crucial because, unlike cataract patients, the RLE patient population already has excellent quality of vision. To mirror the high success rate of contact lens monovision, the key prerequisites will be achieving outstanding distance vision in one eye, and customizing the amount of myopia in the near eye. Because it would be reversible, the risk of intolerable anisometropia would be mitigated. Unlike with keratorefractive surgery, the benefits will not later be lost due to cataracts; indeed, patients will enjoy knowing that they will never develop cataracts in the future. If adjustable EDOF optics can achieve spectacle independence with mini-monovision while minimizing dysphotopsia and maximizing optical quality, this could easily become the most popular presbyopia correcting strategy for both cataract and RLE patients.

\section{Disrupting simultaneous bilateral surgery and global refractive IOL adoption}

I predict that many adjustable IOL patients will elect to have bilateral sameday sequential surgery, just as most patients now do with LASIK. Thanks to shrinking complication rates, bilateral same day cataract surgery is gaining in popularity in many countries and settings where there is no financial disincentive to operating on both eyes simultaneously. One benefit of staging surgeries separately has been the ability to alter the refractive game plan after the first eye results become apparent to the patient and surgeon. For example, many myopic patients coveting excellent uncorrected distance vision become dismayed to find postoperatively how often they must don readers to see anything close. Sequentially staged surgery allows us to target some myopia in the second eye. It also allows the surgeon to make compensatory adjustments to IOL power selection following a refractive surprise in the first eye.

Adjustability would solve both of these problems. Furthermore, simultaneous bilateral adjustable IOL surgery allows patients to immediately experiment with different binocular refractive strategies-e.g., bilateral distance or low myopia, mini-monovision, true monovision, and which is the preferable "distance" eye. Conveniently for patients, optometrists, and ophthalmologists, the postoperative counseling, refractions, adjustments, lock-ins, and examinations could all be simultaneously performed for both eyes. This would also shorten the period that UV protecting glasses would need to be worn. Busy patients will value the reduction in office visits while also avoiding the intervening visual imbalance or anisometropia that accompanies sequentially staged cataract procedures. The refractive premiums should more than offset any reduction in insurance reimbursement.

Aside from several smaller international markets, the premium refractive IOL channel is unique to the US because our healthcare regulatory system properly differentiates between the refractive and medical aspects of cataract surgery. American patients gain access to available refractive technologies because they are allowed to pay for non-medically necessary services, without simultaneously sacrificing their healthcare benefits. One regulatory inconsistency, however, is that patients desiring monofocal 
monovision cannot be billed for refractive services despite requiring just as much preoperative evaluation, counseling, and testing as a presbyopia correcting IOL patient.

From the standpoint of international healthcare regulations and patientshared billing, one benefit of adjustable IOLs is the obvious and legitimate separation of the refractive treatment from the cataract operation. Analogous to LASIK for a pseudophake, the IOL refractive adjustment is not medically necessary and does not take place in the operating room at the same time as the cataract surgery. This LASIK-like model should finally unlock global markets where government health plan restrictions have constrained patient access to premium refractive IOLS. This is typically because the patient must entirely forgo their covered health benefits to obtain a premium IOL privately outside the system.

\section{Addressing the future manpower shortage in eye care}

As the baby boomer generation ages further, a significant manpower shortage in ophthalmology will require ophthalmologists to work closely with optometrists. Adjustable IOLs will provide a perfect opportunity for such collaboration because optometrists embrace and are ideally suited for something that most surgeons would prefer to delegate-patient refractive counseling. Optometrists already excel at monovision contact lens fittings because they can patiently spend the chair time necessary to employ trial and error with different lens powers. If a pseudophakic patient wants to test and compare multiple options postoperatively using trial frames or soft contact lenses, then the optometrist can charge refraction fees commensurate with the amount of time spent. The optometrist will then send the patient back to the surgeon for their postoperative IOL adjustment along with the target refractions that the patient has selected.

This model not only relieves cataract surgeons of the primary refractive counseling responsibility, but allows optometrists to be recognized and appreciated by the patient for their essential role in the overall process. Happy refractive patients will be eternally grateful to the optometrist that took the time to explain and demonstrate the best options. This in turn will cause optometrists to favor and recommend the adjustable IOL modelboth because of the ability to do the refractive counseling postoperatively, and because of the superior refractive outcomes that non-adjustable IOLs cannot match. Finally, the patient-centered benefit of simultaneous bilateral cataract surgery with adjustable IOLs will also improve the efficiency and capacity of optometrists and ophthalmologists to meet our population's expanding need for cataract and IOL-based refractive surgery. Increasing global adoption of RLE will also reduce the cataract surgical burden for future generations of ophthalmologists and for the healthcare system as a whole.

\section{Why adjustable IOLs will be a disruptive innovation}

In summary, adjustable IOLs will deliver multiple new benefits to all three stakeholders-patient, cataract surgeon, and referring optometrist. It is not just the IOL technology that will change, but also the process by which patients will choose their refractive objective, and how optometrists and surgeons will deliver it. Most refractive counseling will shift from preop to postop, with cataract surgeons delegating this important but timeconsuming task to optometrists. The smaller incremental gains afforded by advances in biometry, IOL formulae, digital intraoperative alignment systems, and intraoperative aberrometry could be rendered superfluous by postoperative IOL adjustability. Because of better refractive accuracy, this technology should immediately become the best toric IOL, the preferred monofocal IOL, and the most popular presbyopia correcting strategy.

Patient satisfaction will soar to new heights. After previewing and comparing different refractive outcomes, some pseudophakic patients may prefer being -1.00 in each eye, while others will want mini-monovision with EDOF optics. With respect to the value proposition, they will not need to fully comprehend what astigmatism is. They can simply observe using trial lenses what their vision would be with or without correcting it. Finally, knowing that they can more knowledgeably participate in the decision by comparing different options postoperatively will alleviate the confusion and anxiety that so many of our patients now experience preoperatively.

Observing the obvious refractive benefits and accuracy, referring optometrists will embrace this model and recommend adjustable IOL technology to their patients before they even visit the surgeon. Surgeons will be relieved at no longer having to guess which patients will love a diffractive multifocal and which ones will hate it. And patients who experience the "wow" of their adjustment will better understand the separate value of adjusting and customizing their "prescription" postoperatively, and will champion the technology to their friends.

Untapped global markets for premium IOLs will be unlocked by adjustable IOL technology. In addition to the refractive lens exchange market, clear differentiation between the surgical treatment of cataract and the optional refractive enhancement will allow millions of international cataract patients to access refractive IOL technology and services that their healthcare system currently prevents. Finally, adjustable IOLs will help ophthalmology meet the rising cataract surgical demand by fostering bilateral simultaneous surgery and the delegation of virtually all perioperative refractive counseling to optometrists. Patients will be the biggest winners thanks to better refractive outcomes than we have ever provided, and the ability to experience and choose what refraction they want before making a decision to target and pay extra for it. $\square$ 